\title{
Creatividad en la infancia temprana. Análisis cualitativo en un contexto educativo
}

Laura López Megías. Universidad de Granada

Antonio Fernández-Castillo. Universidad de Granada

Recepción: 9.01.2018| Aceptado: 19.02.2018

Correspondencia a través de ORCID: Antonio Fernández

0000-0002-4362-7650

Citar: Fernández-Castillo, A. y López-Megías, L. (2018). Creatividad en la infancia temprana. Análisis cualitativo en un contexto educativo. ReiDoCrea, 7, 43-54.

Resumen: Aunque la creatividad en la infancia temprana es un asunto de alto interés para la psicología evolutiva y de la educación, no existen demasiados estudios centrados en su evaluación y descripción, debido a las dificultades metodológicas que ello plantea. Objetivos: El objetivo de este trabajo es un análisis exploratorio de la creatividad en educación infantil. Adicionalmente se han estudiado posibles diferencias en función del género, características evolutivas y otros aspectos de interés. Método: En el estudio participaron un total de 80 alumnos del segundo ciclo de educación infantil (41 niños y 39 niñas). Como instrumentos se consideraron dibujos infantiles y entrevistas posteriores a cada participante. Los datos obtenidos fueron analizados en base a diferentes indicadores creativos. Resultados: Los resultados obtenidos ponen de manifiesto el gran potencial creativo en la infancia temprana, así como la tremenda utilidad de los instrumentos utilizados para evaluar las variables objeto de estudio. Conclusiones: El uso de metodologías de análisis cualitativo como alternativa de evaluación, permite acceder a un análisis de la creatividad en la infancia temprana, ámbito donde además no existen demasiados instrumentos estandarizados o con suficiente validez y fiabilidad. Se discuten implicaciones evolutivas y aplicadas en el contexto educativo infantil.

Palabras clave: Creatividad | Infancia

\section{Creativity in Early Childhood. Qualitative Analysis in an Educational Context}

Abstract: Although creativity in early childhood is a matter of high interest for developmental and educational psychology, there are not many studies focused on its evaluation and description, due to the methodological difficulties that this implies. Objectives: The objective of this study is an exploratory analysis focused on creativity in early childhood education. In addition, possible differences between genders, developmental traits and other aspects of interest were studied. Method: The study involved a total of 80 students of second cycle of early childhood education ( 41 boys and 39 girls). As instruments, the children's drawings and interviews to each participant were considered. The data obtained were analyzed based on different creative indicators. Results: The results obtained show the great creative potential in early childhood, as well as the tremendous utility of the instruments used to evaluate the variables under study. Conclusions: The use of qualitative analysis methodologies as an evaluation alternative allows creativity in early childhood to be analyzed, an area where there are not many standardized instruments or with enough validity and reliability. Developmental and applied implications in the early educational context are discussed.

Keywords: Creativity | Childhood

\section{Introducción}

La creatividad ha sido a lo largo del tiempo un tema muy estudiado, su amplitud es la razón de que gran número autores difieran en la definición de este concepto. Si bien este término se ha relacionado comúnmente con el arte, la actividad plástica y la artesanía, abarca muchos más campos y dimensiones (Durán, Esteban Abengozar, Magallón, Martire, Rebouças y Weixlberger, 2013). Inicialmente se relaciono además de con el arte con la teología y la filosofía pero en el siglo XIX la creatividad comienza a ser asociada con campos como la ciencia, la tecnología, etc. (Chavarría, 2015). 
La mayoría de los investigadores coindicen en que la creatividad implica el desarrollo de un producto, idea o solución de un problema de valor para la sociedad (Hennessey y Amabile, 2010).Para algunos autores como Kraft (2005) la creatividad surge de combinar los conocimientos previos con las ideas nuevas para producir resultados originales.

Para hablar de creatividad los autores inciden en el valor del producto final de este proceso. Para Regadera López y Sánchez Carrillo (2015) no basta con la capacidad de crear, si la idea obtenida carece de valor para la sociedad. Se espera un resultado útil, eficaz y práctico.

En numerosas ocasiones podremos encontrar la idea de creatividad relacionada con conceptos como la innovación y el aprendizaje (Fernández, Eizagirre, Arandia, Ruiz de Gauna y Ezeiza, 2012). La innovación implica el uso de la creatividad y ambos términos suelen diferenciarse considerando la creatividad como el desarrollo de las ideas y la innovación como la puesta en práctica de estas (Chavarría, 2015).

Estudiando las definiciones de algunos expertos las similitudes son notables, así por ejemplo Granado (2005, citado en Regadera López y Sánchez Carrillo, 2015, p.10) afirma "Un niño es creativo cuando posee una capacidad superior para producir, visualizar, dramatizar e ilustrar un concepto, una idea o un producto nuevo".

Sternberg (1992, citado en Regadera López y Sánchez Carrillo, 2015, p.10) se basa en el término "insight" para definir la creatividad. "Una especie de intuición que tras un cierto tiempo observando el problema o la situación planteada, consigue, mediante un chispazo creativo, encender la luz que alumbra la solución correcta". Por lo tanto para este autor la creatividad se manifiesta como la capacidad de hallar una estrategia para resolver una situación. Y destaca tres facetas que influyen en la persona creativa: la inteligencia, el estilo intelectual y la personalidad.

Goleman (2002, citado en Regadera López y Sánchez Carrillo, 2015, p.10) expone: "La creatividad es una suerte de adaptación a las circunstancias. Una persona profundamente adaptada a las condiciones que encuentre será muy creativa".

Para Vigotsky (2003) la creatividad haría referencia a cualquier actividad humana que implicase crear algo nuevo. Mientras que para Csikszenmihalyi (1996) la atención es uno de los principales elementos que influyen en la creatividad. Para este autor el proceso creativo conlleva la modificación en un campo simbólico, y esta modificación solo puede darse con la atención suficiente para dominar previamente los conocimientos de dicho campo.

La capacidad de obtener una idea o una solución a determinado problema de manera repentina es valorada como pensamiento creativo, sin embargo los expertos han diferenciado otro tipo de capacidad creativa basado en actitudes como la atención, la disciplina, la apertura-cierre cognitivo o la perseverancia (Hazir y García-Ramírez, 2015; Zeibig, 2015).

Estas diferencias han llevado a los expertos a diferenciar dos tipos de pensamiento. El pensamiento divergente y el convergente. El primero se describe como aquel que trata de producir el mayor número de soluciones posibles y selecciona una de estas para resolver un problema concreto. Los expertos atribuyen mayor creatividad a este pensamiento por ser el que busca algo nuevo y construye criterios de originalidad. Las ideas relacionadas con el pensamiento divergente pueden darse de manera 
inesperada en cualquier situación de la vida cotidiana (Álvarez, 2010; Montoya, 2012; Zeibig, 2015).

Continuando con lo recogido por estos autores el pensamiento convergente es considerado completamente racional. No se relaciona tanto con la novedad ya que se basa en analizar la información de la que disponemos, una y otra vez, para facilitar su comprensión y perfeccionar el dominio de los conceptos. Se asocia con la fase práctica del proceso creativo. Si bien algunos expertos restan importancia al pensamiento convergente en lo referente al proceso creativo, hoy en día numerosos autores atribuyen importancia a ambos pensamientos, por ser tanto el convergente como el divergente considerados esenciales en el desarrollo de nuevas ideas.

Además de tipos de pensamiento podemos diferenciar niveles de creatividad. Según Irving A. Taylor (citado en Casas Carbajo, 2000) encontramos cinco niveles:

1. Creatividad expresiva, se relaciona con la fantasía e improvisación del individuo. Se caracteriza más por la espontaneidad que por la originalidad.

2. Creatividad productiva, por la que se obtienen nuevos productos a partir de un tema determinado.

3. Creatividad inventiva, tiene igualmente como resultado un producto nuevo pero el sujeto no está condicionado externamente.

4. Creatividad innovadora, que se da cuando junto a la innovación se da algo nuevo dejando obsoletos los productos anteriores.

5. Creatividad emergente, consiste en las producciones del sujeto con aportaciones nuevos y bajo el dominio de técnicas creativas.

No existe duda del importante papel que juega el proceso creativo en la sociedad actual, la cual sufre una constante evolución. Esto nos obliga a la continua actualización de los conceptos así como a preparar a las personas para adaptarse a estos cambios (Casas Carbajo, 2000).

La creatividad es uno de los rasgos que poseen las personas desde su nacimiento, por lo tanto todos los seres humanos son creativos por naturaleza, siendo una capacidad que puede ser cultivada y estimulada(Marín, 1991; Ozden, 1993, citado por Erdogdu, 2006).

Este hecho pone en evidencia que el proceso creativo debe verse reflejado en el sistema educativo, sin embargo la importancia de la creatividad no ha sido considerado hasta recientemente. Generalmente los enfoques educativos convencionales han tratado de desarrollar el pensamiento convergente, pero los enfoques actuales se proponen impulsar las capacidades creativas y el pensamiento divergente en los individuos (Beltrán, Garzón y Burgos, 2016; Yildirim, 2010).

Esto se debe a que la metodología tradicional espera una respuesta concreta a un determinado problema conduciéndonos en algunas ocasiones a la frustración del alumno, sin embargo una metodología creativa ofrece mayor variedad tanto en los problemas como en las soluciones (Cemades, 2008).

Según Romero (2010) el sistema educativo actual, el currículo de educación, los materiales, el funcionamiento de los centros, el método evaluativo y la actuación docente, precisan de un cambio que los aproxime a la innovación, a la metodología flexible, y por lo tanto, al concepto de creatividad. 
La etapa preescolar es fundamental en este sentido pues el momento más destacado en el desarrollo de la actitud creativa. El juego cobra un papel fundamental en este proceso por ser la estrategia más adecuada para el niño en cuanto al desarrollo de la capacidad creativa se refiere (Lebrero y Lebrero, 1991).

Casas Carbajo (2000, p. 50) basándose en el estudio de Weisberg y Springer recoge algunas de las tendencias que presentan los niños creativos:

1. Suelen dar respuestas no convencionales

2. Tienen buena capacidad de abstracción

3. Relaciona con facilidad unos hechos con otros

4. Tiene un autoconcepto positivo

5. Tiene buena memoria a corto plazo

6. Tiene sentido del humor

7. Es imaginativo y fantástico

8. Tiene sensibilidad estética

Referente de este mismo modo a criterios que nos permitan evaluar la creatividad, Khatena y Torrance (1976) hablan de cuatro factores, siendo estos los siguientes:

1. Fluidez: La cantidad de respuesta o ideas que ofrece el individuo ante un problema concreto.

2. Flexibilidad: La variedad y clasificación de dichas respuestas.

3. Elaboración: Se relaciona con la sensibilidad del individuo y su capacidad de añadir detalles a la respuesta principal.

4. Originalidad: La calidad de las respuestas en función de ofrecer soluciones poco convencionales.

El individuo experimenta trasformaciones respecto a su capacidad creativa. Algunos autores coinciden en la idea de que las capacidades creativas aumentan a medida que progresa el desarrollo psicosocial (Domínguez, Díaz-Pereira, y Martínez-Vidal, 2015).

Para Csikszentmihalyi (1996) la creatividad se mantiene con la edad tanto en lo referente a la cantidad como a la calidad.

Según Vigotsky (2003, p.17) "cuanto más rica sea la experiencia humana, tanto mayor será el material del que dispone esa imaginación. Por eso, la imaginación del niño es más pobre que la del adulto, por ser menor su experiencia".

Atendiendo a diferencias de género, no existe unanimidad en las opiniones de los investigadores. Así, mientras que por ejemplo Donolo y Elisondo (2007) afirman que las diferencias entre ambos sexos no son significativas, otros estudios más recientes (Chiecher, Elisondo, Paoloni y Donolo, 2018) han encontrado evidencia que apunta a mayores niveles de creatividad en mujeres universitarias.

A pesar de la relevancia del tema, de las grandes implicaciones que implica para la actividad educativa (Elisondo, 2015) y del interés que suscita en ámbitos académicos y científicos, no demasiados trabajos han estudiado la creatividad en la infancia temprana. Quizá ello se deba a la dificultad de evaluar o determinar las manifestaciones de la creatividad a estas edades tan tempranas. $Y$ aun son menos las investigaciones que se han centrado en estos tópicos desde la perspectiva de los propios sujetos. 


\section{Objetivos}

El objetivo general de este trabajo es analizar la creatividad en la infancia temprana. Como objetivos específicos nos proponemos en primer lugar explorar la expresión del comportamiento creativo e innovador desde el punto de vista infantil y a través del dibujo. En segundo lugar nos planteamos estudiar los aspectos señalados desde una perspectiva evolutiva, es decirla posible evolución de los aspectos anteriores a lo largo de la infancia temprana, concretamente pretendemos analizar posibles diferencias entre niños de 3, 4 y 5 años de edad. Finalmente, en tercer lugar nos proponemos explorar diferencias de género en la expresión de la creatividad en la infancia temprana.

\section{Método}

Para alcanzar los objetivos planteados en este estudio se empleo una metodología cualitativa descriptiva con un análisis del contenido posterior. Como técnica específica de recogida de datos se utilizó el dibujo infantil dirigido a obtener un punto de vista subjetivo sobre el objeto de estudio. Por tratarse de participantes de corta edad y por ocasionales dificultades en la interpretación de los dibujos, se utilizó adicionalmente una entrevista no estructurada para obtener información adicional y explicaciones sobre los dibujos planteados.

\section{Participantes}

El estudio se ha llevado a cabo en un colegio privado-concertado de Educación Infantil de la provincia de Granada entre tres clases de educación infantil de primer, segundo y tercer curso.

Han participado un total de 80 alumnos, de los cuales 41 eran niños y 39 eran niñas. Respecto a la edad el rango oscila entre los 3 y los 6 años de edad $(M=4.26$; $\mathrm{DT}=0.88)$. De forma más específica colaboraron 18 alumnos de tres años, 28 alumnos de cuatro años, 29 alumnos de cinco años y cinco alumnos de seis años de edad.

En lo referente al ámbito familiar predomina un nivel socio-cultural y económico alto. La mayoría de padres disponen de formación académica y están empleados actualmente. Culturalmente no hay diferencias considerables entre los alumnos.

\section{Instrumentos}

Los instrumentos empleados para la recogida de datos de este estudio han sido dibujos y entrevistas.

Se les pidió a los niños y niñas la realización de un dibujo recibiendo previamente la instrucción: "Dibújate a ti mismo inventado algo". Se aplico este método ya que el dibujo es considerado como una forma de expresión infantil en la que los niños reflejan de manera válida sus sentimientos, emociones, intereses, miedos y deseos (Molina, 2015). Es considerada una forma de comunicación ya que representa de forma no verbal la visión que el niño ha elaborado del mundo (Puleo, 2012). Cabe destacar la relevancia del dibujo en este estudio concreto ya que éste contribuye al desarrollo de la creatividad e imaginación en los niños preescolares (Sánchez, 2015). Tras finalizar sus dibujos y de forma individual se les pregunto a cada niño qué había dibujado para identificar cada uno de los elementos plasmados. 


\section{Procedimiento}

Para llevar a cabo el estudio en primer lugar se consultó con la dirección y los docentes del centro correspondientes a las clases de primero, segundo y tercer ciclo para obtener conformidad y autorización. Adicionalmente se solicitó a través de los docentes autorización parental a las familias para proceder con la recogida de datos. Se explicó a los padres que en ningún caso se recopilarían datos ni información que permitiese identificar a los participantes y se obtuvo consentimiento informado firmado.

Los dibujos se realizaron en diferentes fechas durante un margen de un mes para todos los cursos y en todos los casos en condiciones similares.

Comenzamos en la clase de primer curso, correspondiente a alumnos de tres y cuatro años de edad, el día 12 de abril de 2016. Tras el periodo de recreo los alumnos se relajaron en la mesa mientras sonaba música clásica y aproximadamente 20 minutos más tarde de la entrada del patio de recreo, a las 12:20, la música se paró y se les entregó un folio en blanco y ceras de colores a los alumnos, los cuales están organizados en grupos de entre 4 y 6 niños. Se les explicó que debían hacer un dibujo y se les dio la instrucción citada anteriormente. Este procedimiento se llevo a cabo de forma similar en las aulas de segundo y tercer curso.

El dibujo se realizo en el aula de segundo curso el día 5 de mayo de 2016 a alumnos de cuatro y cinco años. La relajación ya se había llevado a cabo cuando entré en el aula y el procedimiento para realizar el dibujo fue exactamente el descrito anteriormente.

Por último en el aula de cinco años la realización del dibujo tuvo lugar el día 11 de mayo de 2016. De igual manera que en el caso anterior en primer lugar el docente del aula me presentó a los alumnos. Tras la relajación les expuse el enunciado para la realización del dibujo. Estos alumnos disponían de mayor variedad de materiales en comparación a las aulas anteriores, como rotuladores y lápices de colores.

En todas las aulas el orden que se llevó a cabo para la realización de las entrevistas correspondió al orden en el que cada alumno termino su dibujo. El tiempo para realizar el dibujo era libre y la media en la realización fue de unos 15 minutos, excepto en el aula de cinco años donde los alumnos emplearon mayor tiempo de media para finalizar sus dibujos. En las tres situaciones, se encontraban en el aula las docentes correspondientes a dichos alumnos, las cuales no intervinieron en el proceso que se realizó para este estudio. La instrucción "Dibújate a ti mismo inventando algo" se repitió en varias ocasiones a lo largo de la realización de los dibujos.

Finalmente los dibujos se analizaron atiendo a las diferencias de género, masculino y femenino, y edad, en la que se dispusieron tres categorías, correspondientes al año de nacimiento del alumno y por lo tanto al curso escolar en el que se encuentra, diferenciando entre alumnos de 3,4 y 5 años de edad.

\section{Análisis de datos}

En el estudio realizado se ha puesto en práctica un análisis de contenido para la extracción de los resultados. Para dicho análisis de contenido se tuvieron en cuenta diversos criterios de categorización y codificación y como unidad de análisis se optó por considerar una técnica de libre flujo. Adicionalmente nos hemos fijado en aspectos formales y estructurales. Para el establecimiento categorial, se buscaron patrones 
repetitivos y pautas así como aspectos diferenciales y llamativos de la percepción de los participantes.

\section{Resultados}

En este trabajo nos proponíamos analizar la capacidad creativa e inventiva con una muestra de niños de educación infantil. En relación con el primero de nuestros objetivos, en el que nos planteábamos examinar la expresión del comportamiento creativo desde el punto de vista del alumno mediante el dibujo, nuestros resultados han mostrado números rasgos de creatividad y respuestas no convencionales a destacar, como se puede apreciar en las figuras 1 y 2 .

\section{Figura 1: Ejemplo de respuesta creativa original "Cachivache para bucear"}

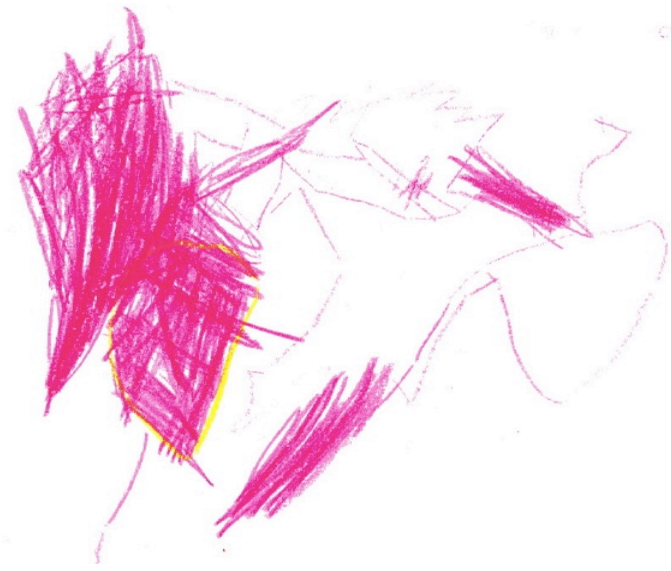

Figura 2: Ejemplo de respuesta "Máquina que coge a los niños para subirlo al tobogán y desprende globos robots"

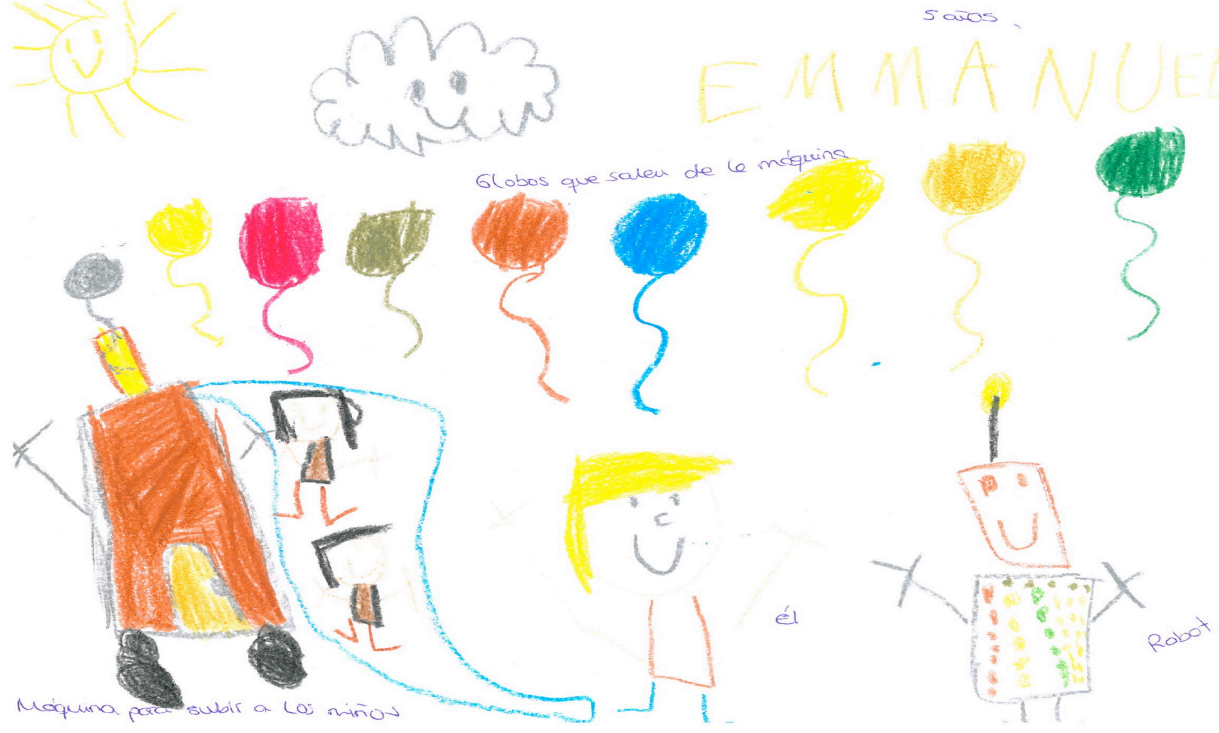

Atendiendo a nuestros siguientes objetivos, como eran el análisis de diferencias respecto a la capacidad creativa en referencia al sexo y edad, los resultados han mostrado lo siguiente: En primer lugar los alumnos de 3 y 4 años han presentado mayor dificultad para comprender la instrucción que se les dio, el $84 \%$ y $83 \%$ respectivamente se han dibujado a ellos mismos como se les indicó, frente al $100 \%$ de los alumnos de 5 años. Además, el $27 \%$ de los niños de 3 años dibujaron también a 
uno o varios miembros de su familia. En el caso de los alumnos de 4 y 5 años para ambos grupos este resultado es del $17 \%$. De igual modo, la segunda parte de la instrucción, que hacía referencia a que se dibujasen inventando algo está en menor medida de forma considerable entre los dibujos de 3 y 4 años. Algunos elementos comunes entre todos dibujos son que el alumno se dibuje sonriendo, la presencia de elementos de la naturaleza (flores, arboles, nubes, sol), dibujar animales y representar elementos animistas. Los elementos a destacar como innovadores entre los dibujos del grupo de tres años se relacionan en su mayoría con actividades físicas o elementos ficticios. Algunos de los dibujos han sido realizados con un solo color y son escasos en detalles. Algunas de estas características se observan en la figuras 3 y 4.

Figura 3: Ejemplo de dibujo "Los círculos de colores se colocan en la cabeza y el cabello cambia de color"

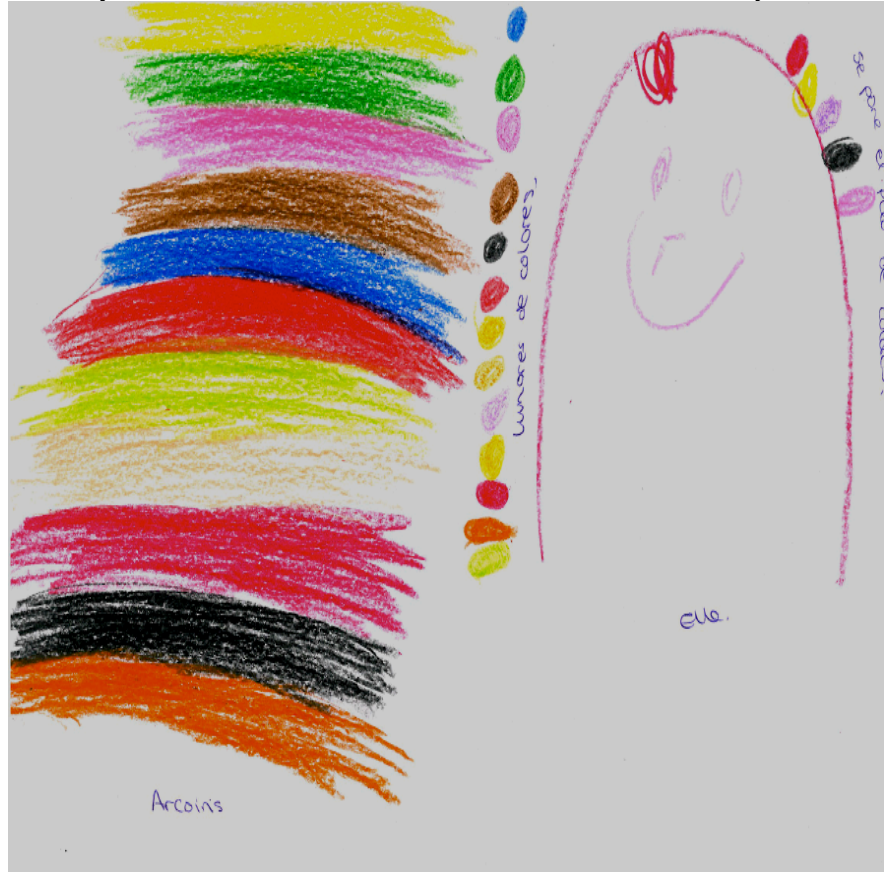

Figura 4: Ejemplo de dibujo sobre actividad infantil "Cuerda para colgar en el cielo y que los niños se tiren"

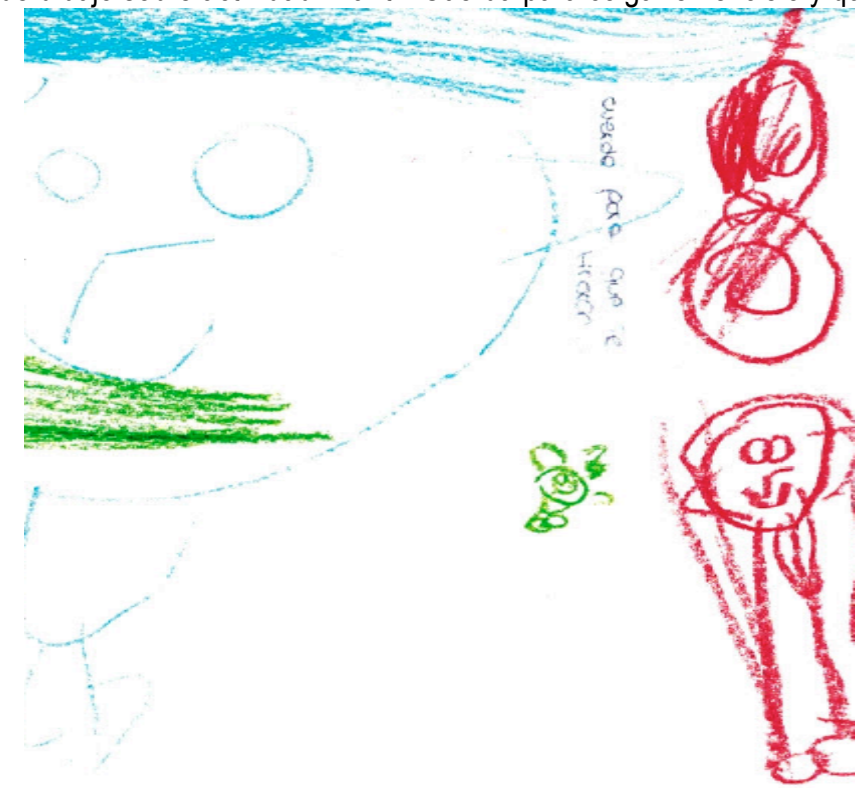


Continuando con el grupo de tres años los elementos dibujados son repetidos con frecuencia entre los alumnos y son escasos los que presentan componentes originales. Predomina mayor número de garabatos y de elementos que el propio alumno que los ha dibujado no sabe identificar que en los grupos de cuatro y cinco años.

En el grupo de cuatro años predominan elementos de construcción, 12 de los 23 participantes han representado cabañas, casas del árbol o castillos. También cabe destacar la presencia de personajes o escenarios de dibujos animados mediáticos. Observamos en estos dibujos mayor cantidad de elementos y mayor superficie del folio ocupada por el dibujo en comparación al grupo anterior. No hay ningún dibujo monocromático.

Por último entre los dibujos de los alumnos de cinco años destacamos numerosos elementos de carácter innovador y de gran variedad. Predominan objetos electrónicos como ordenadores, auriculares y robots. Todos los niños se han dibujado realizando alguna actividad, jugando o utilizando algún elemento. Aparecen gran variedad de elementos y detalles, los colores se corresponden con la realidad y todos emplean una amplia gama de colores. Son ejemplos ilustrativos de estos elementos los dibujos incluidos en las figuras 5 y 6 .

Figura 5: Ejemplo de dibujo sobre objetos electrónicos "El niño tiene una bombilla en la cabeza porque ha tenido la idea de crear al robot, el cual sirve para hacer carreras y da trapos para el sudor"

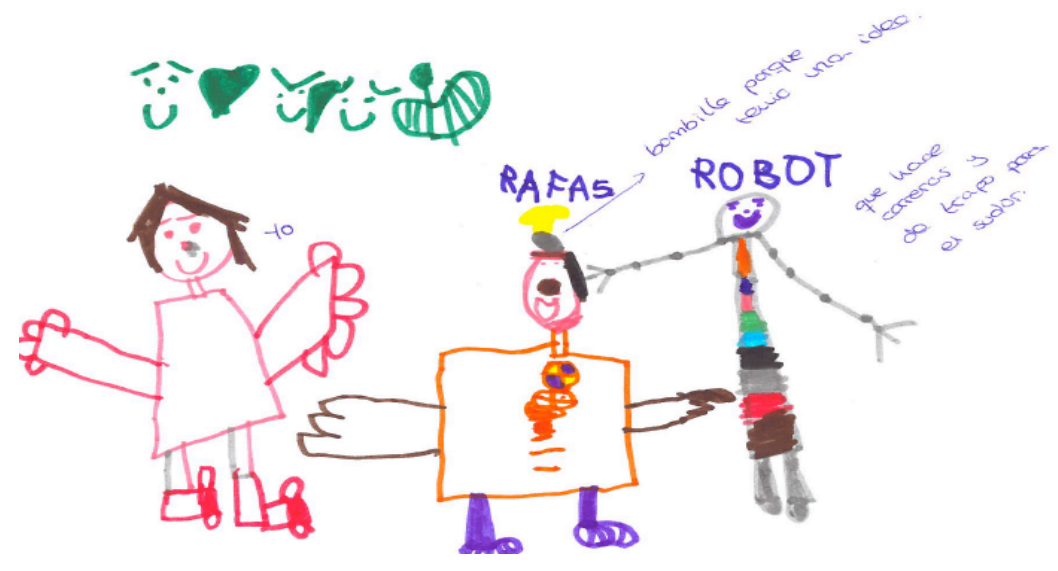

Figura 6: Ejemplo de dibujo sobre máquinas y objetos electrónicos "Maquina en la que se introduce una cosa y salen cinco"

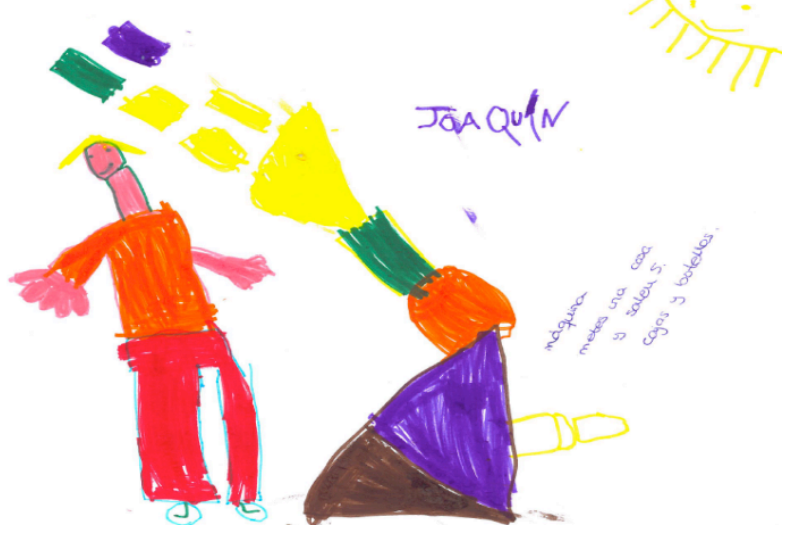


En relación con el último de nuestros objetivos y posibles diferencias atendiendo al género, la más significativa es la evidencia sobre la variedad de color, siendo las niñas las que emplean más colores, aunque esta diferencia va disminuyendo en los grupos de mayor edad. Igual pasa con la cantidad de elementos y detalles en las que las niñas conforman un porcentaje más alto, especialmente en el grupo de menor edad. Sin embargo, teniendo cuenta los dibujos que reflejaban elementos de innovación, es mayor el número de niños que de niñas que ha representado estos elementos.

A modo de resumen, en la tabla 1 se esquematiza un breve análisis de las principales categorías en las que se pueden acotar nuestros resultados, y que han sido descritos a lo largo de este apartado.

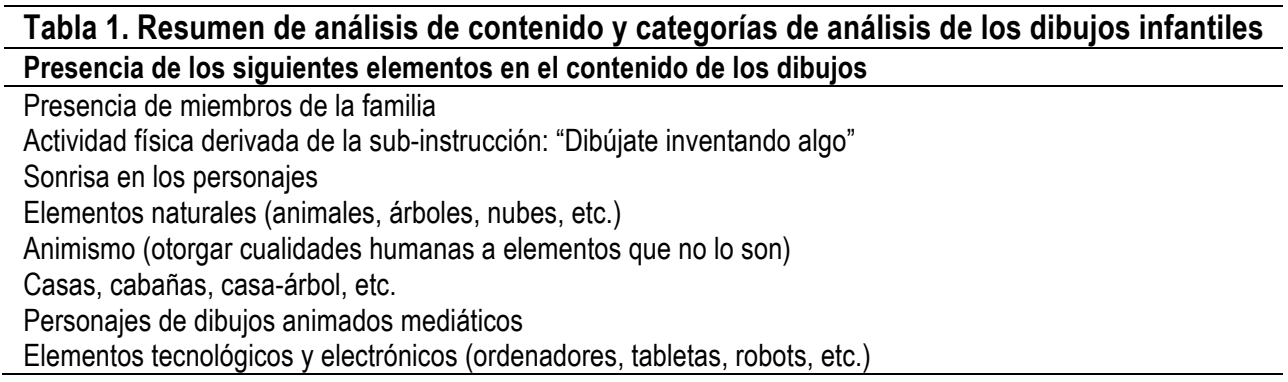

\section{Discusión}

Tras concluir el análisis de datos y evaluar los resultados, hemos podido constatar que los criterios de creatividad, tales como fluidez, flexibilidad, elaboración y detalle, están presentes en la educación temprana y el dibujo es un cauce en el que estos elementos se ponen de manifiesto. Además, los dibujos incluyen un derroche de imaginación que se representa en las actividades de los personajes, los elementos secundarios o las incorporaciones de inventos generados por los participantes.

En el análisis de los dibujos de este estudio hemos podido observar las tendencias características de niños creativos señaladas por Casas Carbajo (2000), además de poder evaluar el nivel creativo general del grupo basándonos en los indicadores de Khatena y Torrance (1976). En general y casi en la totalidad de los dibujos se observa improvisación y expresión de fantasía, creatividad productiva y emergente. En la medida en que se ha utilizado una técnica que favorece la expresividad en la infancia temprana como es el dibujo, los participantes han producido expresiones novedosas y divergentes.

De tal modo, las respuestas menos convencionales se han mostrado en mayor medida en el grupo de cinco años. De la misma forma destacan las respuestas del grupo de tres años y en menor medida el de cuatro años.

Respecto a la fluidez y elaboración, es también el grupo de cinco años el que destaca por estos factores, como era de esperar. Los detalles, el número de elementos, el uso de colores, en definitiva, la sensibilidad estética va evolucionando con el crecimiento del niño y es fácilmente apreciable al comparar los dibujos.

Se ven reflejadas también en este estudio las ideas de Vigotsky (2003) referidas a la experiencia personal y la creatividad, pues a pesar de que los alumnos de cinco años han sido los que más respuestas creativas han proporcionado también son los que más se ajustan a la realidad y dominan nuevos campos. 
Al hablar de las diferencias de género, los resultados de este estudio no llegan a presentar desigualdades significativas, al igual que el estudio llevado a cabo por Donolo y Elisondo (2007).

Como una posible dificultad para llevar a cabo este estudio podemos mencionar la hora de la que se disponía para pedirles a los alumnos la realización dibujo, ya que al ser tras el tiempo de recreo los niños están cansados y ya han realizado actividades de dibujo.

Una de las limitaciones de nuestro estudio ha estado en la dificultad encontrada en algunos de los participantes para seguir la instrucción dada para llevar a cabo el dibujo. Para próximos estudios sería recomendable que el tema sea introducido a los alumnos de forma previa a la realización del dibujo, lo que facilitaría la comprensión de la instrucción y el objetivo del dibujo en sí. Ello enriquecería los datos a obtener.

Futuras investigaciones podrán aprovechar la metodología utilizada aquí para evaluar la creatividad en la infancia, ya que se incorpora un procedimiento que se ha mostrado eficaz con sujetos en los que otras pruebas de evaluación basadas en lenguaje más complejo podrían no resultar operativas. La relación de la creatividad con otras variables y aspectos psicológicos en la infancia temprana, pueden ser también sugerencias a tener en cuenta para futuras líneas de investigación.

Para finalizar, insistir en la importancia de fomentar la capacidad creativa en la infancia temprana en el contexto educativo. Sin lugar a dudas no se deben escatimar esfuerzos para dotar al sistema educativo de los medios materiales y humanos que permitan el impulso de la creatividad en cualquier faceta de este ámbito. Las nuevas sociedades en todos los ámbitos de su desarrollo demandan eminentemente personas creativas, siendo en la infancia donde esta capacidad parece expresar los mayores niveles de funcionamiento tanto en un nivel cuantitativo como cualitativo. Partiendo de esta premisa el trabajo en la infancia temprana parece crucial al ser un periodo determinante para el desarrollo de la creatividad.

\section{Referencias}

Álvarez, E. (2010). Creatividad y pensamiento divergente. Desafío de la mente o desafio del ambiente. Archived by WebCite ${ }^{\circledR}$ at http://www.webcitation.org/6wJckr5pw.

Beltrán, CY., Garzón DM. y Burgos, NC. (2016). Incidencia del fortalecimiento del pensamiento divergente en la creatividad de los niños. Infancia Imágenes, 15(1), 103-118.

Casas Carbajo, J. (2000). La creatividad en la Educación Infantil, Primaria y Secundaría. Madrid: EOS.

Cemades, I. (2008). Desarrollo de la creatividad en Educación Infantil. Perspectiva constructivista. Creatividad y Sociedad, 12, 7-20.

Chavarría, MA. (2015). La eficacia de la creatividad: Creactívate. Madrid: ESIC.

Chiecher, AC., Elisondo, RC., Paoloni, PV., y Donolo, DS. (2018). Creatividad, género y rendimiento académico en ingresantes de ingeniería. Revista Iberoamericana de Educación Superior, 9(24), 138-151.
Csikszentmihalyi, M. (1996). Creatividad: el fluir y la psicología del descubrimiento y la invención. Barcelona: Paidós Ibérica.

Domínguez, A., Diaz-Pereira, M. y Martinez-Vidal, A. (2015). The evolution of motor creativity during primary education. Journal of Human Sport and Exercise, 10(2), 583-591.

Donolo, DS. y Elisondo, RC. (2007). Creatividad para todos. Consideraciones sobre un grupo particular. Anales de Psicología, 23(1), 147-141.

Durán, T., Esteban Abengozar, A., Magallón, R., Martire, A., Rebouças, B. y Weixlberger, C. (2013). La creatividad. RUTA: Revista Universitària de Treballs Acadèmics, 5, 1-22.

Elisondo, R. (2015). La creatividad como perspectiva educativa. Cinco ideas para pensar los contextos creativos de enseñanza y aprendizaje. Revista Electrónica Actualidades Investigativas en Educación, 15, 1-23.

Erdogdu, MY. (2006). The adaptation of creativity assessment scale to Turkish culture. Inonu University Egitim Fakultesi Dergisi, 7(12), 61-79. 
Fernández Fernández, I., Eizagirre Sagardia, A., Arandia Loroño, M., Ruiz de Gauna Bahillo, P. y Ezeiza Ramos, A. (2012). Creatividad e innovación: claves para intervenir en contextos de aprendizaje. Revista Iberoamericana sobre Calidad, Eficacia y Cambio en Educación, 10(2), 23-40.

Hazir, M., \& García-Ramírez, JM. (2015). Creativity \& Cognitive Closure. Reidocrea, 4, 18-23.

Hennessey, BA., \& Amabile, TM. (2010). Creativity. Annual Reviews of Psychology, 61, 569-598.

Khatena, J., \& Torrance, EP. (1976). Manual for Khatena-Torrance Creative Inventory. Chicago: Stoelting.

Kraft, U. (2005). Creatividad: Las ideas innovadoras son el resultado de un complicado proceso cognitivo, cuyo mecanismo van descifrando neurólogos y psicólogos. Mente y Cerebro, 11, 42-46.

Lebrero, MP. y Lebrero MT. (1991) Creatividad en la educación infantil. En R. Martín y S. de la Torre (Comps.), Manual de la creatividad. Aplicaciones educativas (pp. 357-360). Barcelona: Vicens Vives.

Marín, R. (1991). Definición de la creatividad. En R. Marín y S. de la Torre (Comps.), Manual de la creatividad. Aplicaciones educativas. (pp. 95-99). Barcelona: Vicens Vives.

Molina, A. (2015). El dibujo infantil: Trazos, colores e historias que nos hacen reflexionar y aprender. Revista Electrónica Educare, 19(1), 167-182.

Montoya, FJ. (2012). La solución de problemas de manera innovadora, exploración de un camino creativo. Revista Trilogía, 7, 125-133.

Puleo, E. (2012). La evolución del dibujo infantil. Una mirada desde el contexto sociocultural merideño. Educere: Investigación Arbitrada, 53, 157-170.

Regadera López, A. y Sánchez Carrillo, JL. (2015). Creatividad: cómo evaluarla y cómo potenciarla. Valencia: Brief Ediciones.

Romero, J. (2010). Creatividad distribuida y otros apoyos para la educación creadora. Pulso, 33, 87-107.

Sánchez Sánchez, RA. (2015, 18 de noviembre). Dibujo como representación mental para desarrollar la función simbólica de los niños de educación inicial. Archived by WebCite ${ }^{\circledR}$ at http://www.webcitation.org/6wJd1mqcf.

Vigotsky, LS. (2003). La imaginación y el arte en la infancia. Madrid: Akal.

Yildirim, A. (2010). Creativity in early childhood education program. Procedia-Social and Behavioral Sciences, 9, 15611565.

Zeibig, D. (2015). Estrategias para un pensamiento creativo. Mente y Cerebro, 70, 36-41. 